\title{
REDUCTION THEOREM FOR CONNECTIONS AND ITS APPLICATION TO THE PROBLEM OF ISOTROPY AND HOLONOMY GROUPS OF A RIEMANNIAN MANIFOLD
}

\author{
KATSUMI NOMIZU
}

The present paper constitutes, together with [13], a continuation of the study of differential geometry of homogeneous spaces which we started in [11]. Our main result states that if the homogeneous holonomy group of a complete Riemannian manifold is contained in the linear isotropy group at each point, then the Riemannian manifold is symmetric. The converse is of course one of the well known properties of a Riemannian symmetric space [4]. Besides the results already sketched in [12], we add a few applications of the main theorem.

After giving a brief sketch of the general theory of connections and holonomy groups, we first establish a reduction theorem for connections in a principal fiber bundle. Although it is just an exact formulation of E. Cartan's theorem [3] and is supposedly "well known" by now, there is no published proof as yet.

By using this theorem, we prove Theorem 1 on certain invariant affine connections on a homogeneous space. Then applying the method developed in [13] and using Theorem 1, we obtain Theorem 2 which is the main result.

I am indebted to Professor Lichnerowicz and to my friend S. Kobayashi for stimulating conversations on the topics treated in this paper.

\section{Reduction of a connection}

1. Connection in a principal fiber bundle. Although the notion of a connection in a principal fiber bundle is well known [1], [5], [7], we shall give a brief resumé of the definitions which we need for our purpose. By differentiability, we shall always understand that of class $C^{\infty}$.

Let $P=P(B, G)$ be a differentiable principal fiber bundle over the base manifold $B$ with structure Lie group $G$ [7], [15]. We always assume that $P$ satisfies the second axiom of countability. Then this is true for the base $B$

Received March 3, 1955. 
and the structure group $G$. For $G$, it means that $G$ is a Lie group (a locally connected group whose identity component $G^{0}$ is an analytic group [6]) which has at most a countable number of components. Conversely, if $B$ satisfies the second axiom of countability and if $G / G^{0}$ is at most countable, then $P$ satisfies the second axiom of countability.

At each point $x$ of $P$, let $P_{x}$ be the tangent space to $P$ and $G_{x}$ the subspace of $P_{x}$ which is tangent to the fiber through $x$. A connection $\Gamma$ in $P$ is a choice of a tangent subspace $Q_{x}$ at each $x$ of $P$ which satisfies the following conditions:

1) $P_{x}=G_{x}+Q_{x}$ (direct sum) ;

2) $Q_{x a}=R_{a} \cdot Q$, where $R_{a}(a \in G)$ denotes the action of $G$ on $P$ to the right, that is, $R_{a} \cdot x=x a$;

3) $Q_{x}$ depends differentiably on $x$.

$Q_{x}$ is called the horizontal subspace at $x$, and a tangent vector of $P$ is called horizontal if it belongs to some $Q_{x}$. The last condition can be explained as follows. Given a vector field $X$ in $P$, we have at each $x \in P$ a unique decomposition $X_{x}=Y_{x}+Z_{x}$, where $Y_{x} \in G_{x}$ and $Z_{x} \in Q_{x}$, by 1$)$. The vector field $Z: x \rightarrow Z_{x}$, called the horizontal component of $X$, is required to be differentiable if $X$ is differentiable. In this case, the vector field $Y: x \rightarrow Y_{x}$, called the vertical component of $X$, is also differentiable.

Given a connection $\Gamma$ in $P$, we define the parallel displacement of fibers along any (piecewise differentiable) curve in $B$. Let $\tau$ be a curve from $u_{0}$ to $u_{1}$ in $B$. Then it can be proved that, for any point $x_{0}$ of $P$ lying over $u_{0}$, there is one and only one curve $\tau^{*}$ beginning at $x_{0}$ which covers $\tau$ and whose tangent vectors are all horizontal (such a curve is called a horizontal curve). We call $\tau^{*}$ the lift of $\tau$ through $x_{0}$. Thus $\tau$ defines a mapping, denoted by the same letter, which maps $x_{0}$ into the end point of the lift $\tau^{*}$ through $x_{0}$. From the condition 2), we have $\tau(x \cdot a)=\tau(x) \cdot a$ for any point $x$ over $u_{0}$ and $a \in G$. It follows that $\tau$ is a differentiable isomorphism of the fiber over $u_{0}$ onto the fiber over $u_{1}$. This is called the parallel displacement along the curve $\tau$.

Let $x_{0}$ be any arbitrary but fixed point of $P$. The set of parallel displacements which correspond to all closed curves at $u_{0}=\pi\left(x_{0}\right)$ form a subgroup $\Psi$ of $G$, which is by definition the holonomy group of the given connection $\Gamma$. Corresponding to all closed curves which are homotopic to zero, we have a sub- 
group $\Psi^{0}$ of $\Psi$, called the restricted holonomy group of the given connection. It is a connected Lie group which is proved to the identity component of $\Psi$. $\Psi$ is a Lie group such that $\Psi / \Psi^{0}$ is at most countable. For the proof, see [1]. We remark that the fundamental group of $B$ is at most countable as it follows from our assumption on the second axiom of countability.

2. Reduction of the structure group. Given two principal fiber bundles $P^{\prime}\left(B^{\prime}, G^{\prime}\right)$ and $P(B, G)$ over the same base manifold $B$, a differentiable mapping $f$ of $P^{\prime}$ into $P$ is called a homomorphism if there exists a homomorphism $f$ of $G^{\prime}$ into $G$ such that $f\left(x^{\prime} a^{\prime}\right)=f\left(x^{\prime}\right) f\left(a^{\prime}\right)$, where $x^{\prime} \in P^{\prime}$ and $a^{\prime} \in G^{\prime}$, and if it induces the identity transformation of the base $B$ onto itself. If it is one-to-one, it is called an isomorphism of $P^{\prime}$ into $P$. In the case where $G^{\prime}$ is a Lie subgroup of $G$, an isomorphism $f$ which corresponds to the injection of $G^{\prime}$ into $G$ is called an injection of $P^{\prime}\left(B^{\prime}, G^{\prime}\right)$ into $P(B, G)$.

The structure group of a principal fiber bundle $P(B, G)$ is said to be reducible to a Lie subgroup $G^{\prime}$ of $G$ if there exists a differentiable principal fiber bundle $P^{\prime}\left(B, G^{\prime}\right)$ and an injection $f$ into $P(B, G)$. In this case, $P^{\prime}\left(B, G^{\prime}\right)$ together with the injection $f$ is called the reduced bundle.

The structure group $G$ of $P(B, G)$ is reducible to $G^{\prime}$ if and only if there exists a suitable covering $\left\{U_{\alpha}\right\}$ of $B$ and an isomorphism of $\pi^{-1}\left(U_{\alpha}\right)$ with $U_{\alpha} \times G$ expressed by $x \in \pi^{-1}\left(U_{\alpha}\right) \rightarrow\left(\pi(x), \varphi_{\alpha}(x)\right) \in U_{\alpha} \times G$ such that the corresponding transit functions $\psi_{\beta \alpha}(u)=\varphi_{\beta}(x) \cdot \varphi_{\alpha}(x)^{-1}, u=\pi(x)$, take their values in the subgroup $G^{\prime}$. It should be remarked that if such a covering and transit functions exist, then $\psi_{\beta \alpha}(u)$ are differentiable mappings from $U_{\alpha} \cap U_{\beta}$ into the Lie subgroup $G^{\prime}$. This may be proved in the same way as Proposition 1, p. 95, of [6]. We can construct the reduced bundle $P^{\prime}\left(B, G^{\prime}\right)$ as follows. For each $\alpha$, consider a space $X_{\alpha}$ which is homeomorphic with $U_{\alpha} \times G^{\prime}$, the homeomorphism being denoted by $g_{\alpha}$. Let $X=\cup_{\alpha} X_{\alpha}$ be the topological sum of $X_{\alpha}$ and introduce an equivalence relation $R$ in $\stackrel{\alpha}{X}$ by

$$
g_{\alpha}\left(u, a^{\prime}\right)=g_{\beta}\left(u, \psi_{\beta \alpha}(u) a^{\prime}\right), \quad u \in U_{\alpha} \cap U_{\beta}
$$

Let $P^{\prime}$ be the quotient space $X / R$ with quotient topology. $G^{\prime}$ acts on $P^{\prime}$ by [class of $\left.g_{\alpha}\left(u, a^{\prime}\right)\right] \cdot b^{\prime}=$ [class of $\left.g_{\alpha}\left(u, a^{\prime} b^{\prime}\right)\right]$. It is easy to verify that $P^{\prime}$ is a differentiable principal fiber bundle $P^{\prime}\left(B, G^{\prime}\right)$ with an obvious injection into $P(B, G)$. 
3. Reduction of a connection. We first prove

Proposition 1. A homomorphism $f$ of $P^{\prime}\left(B, G^{\prime}\right)$ into $P(B, G)$ maps a connection in $P^{\prime}$ into a connection in $P$.

Proof. To establish this, we first define the horizontal subspace at any point of $f\left(P^{\prime}\right)$. Let $f\left(x^{\prime}\right)=x$. We define $Q_{x}$ as the image by $f$ of the horizontal subspace $Q^{\prime}{ }_{x^{\prime}}$ at $x^{\prime}$ of the given connection in $P^{\prime}$. It is independent of the choice of $x^{\prime}$ such that $f\left(x^{\prime}\right)=x$. In fact, if $f\left(x^{\prime}\right)=f\left(y^{\prime}\right)=x$, then $y^{\prime}=x^{\prime} a^{\prime}$ for some $a^{\prime} \in G^{\prime}$ and $Q_{y^{\prime}}^{\prime}=R_{a^{\prime}} \cdot Q_{x^{\prime}}$. We have $f \cdot R_{a^{\prime}}=R_{a} \cdot f$, where $a=f\left(a^{\prime}\right)$ is indeed the identity of $G$ because $f\left(x^{\prime} a^{\prime}\right)=f\left(x^{\prime}\right) f\left(a^{\prime}\right)=f\left(x^{\prime}\right)$ implies that $f\left(a^{\prime}\right)$ $=e$. From this we have $f \cdot Q^{\prime}{ }_{y^{\prime}}=f \cdot R_{a^{\prime}} \cdot Q_{x^{\prime}}^{\prime}=f \cdot Q^{\prime}{ }_{x^{\prime}}$.

For any other point $y$ of $P$, we define the horizontal subspace at $y$ by $Q_{y}=R_{a} \cdot Q_{x}$ if $y=x a$ with $x \in f\left(P^{\prime}\right)$ and $a \in G$ (any point of $P$ is written in such a form). $Q_{y}$ is defined independently of the representation $y=x_{1} a_{1}=x_{2} a_{2}$, where $x_{1}$ and $x_{2}$ belong to $f\left(P^{\prime}\right)$. It is easy to verify that $y \rightarrow Q_{y}$ defines a connection in $P$.

In the case where $f$ is an injection, we say that a given connection $\Gamma$ in $P$ is reducible to a connection $\Gamma^{\prime}$ in $P^{\prime}$ if $f$ maps $\Gamma^{\prime}$ into $\Gamma$ in the manner of Proposition 1.

Now we are in a position to establish

Proposition 2 (reduction theorem for connections). Let $P(B, G)$ be a principal fiber bundle with a connection $\Gamma$, and let $\Psi$ be the holonomy group of $\Gamma$ with reference point at $x_{0}$ of $P$. Then the structure group $G$ is reducible to $\Psi$, and the connection $I$ is reducible to a connection in the reduced bundle $P^{\prime}(B, \Psi)$. Moreover, the reduced bundle may be regarded as a subbundle of $P$ consisting of points which can be joined to $x_{0}$ by a horizontal curve.

Proof. Let $\pi\left(x_{0}\right)=u_{0}$. We construct a covering $\left\{U_{\alpha}\right\}$ of $B$ and a set of transit functions all taking values in $\Psi$. For this purpose, we take any covering $\left\{U_{\alpha}\right\}$ of $B$, each $U_{\alpha}$ being a cube $\left|u^{i}\right|\left\langle\delta_{\alpha}, \delta_{\alpha}>0\right.$, with center $u_{\alpha}=(0,0, \ldots, 0)$ with respect to some local coordinate system $\left(u^{1}, \ldots, u^{n}\right)$. We choose once for all a family of curves $\tau_{\alpha}$ in $B$ all starting from $u_{0}$ and each ending at $\boldsymbol{u}_{\alpha}$. Let $x_{\alpha}=\tau_{\alpha}\left(x_{0}\right)$. Now for any point $x$ in $\pi^{-1}\left(U_{\alpha}\right)$ we take the ray $\rho_{\alpha}$ in $U_{\alpha}$ (with respect to the given local coordinates) from $u=\pi(x)$ to $u_{\alpha}$. We have $\rho_{\alpha}(x)$ $=x_{\alpha} \cdot a$ for some $a \in G$ and we define this element a to be $\varphi_{\alpha}(x)$. Then we 
easily see that $x \rightarrow\left(\pi(x), \varphi_{\alpha}(x)\right)$ gives an isomorphism of $\pi^{-1}\left(U_{\alpha}\right)$ with $U_{\alpha} \times G$. If $x \in \pi^{-1}\left(U_{\alpha} \times U_{\beta}\right)$, a simple manipulation shows that $\psi_{\beta \alpha}(x)=\varphi_{\beta}(x) \cdot \varphi_{\alpha}^{-1}(x)$ belongs to $\Psi$. Namely, we have transit functions all taking values in $\Psi$.

This shows that $G$ is reducible to $\Psi$. The reduced bundle $P^{\prime}(B, \Psi)$, which is constructed from the transit functions $\psi_{\beta \alpha}$ as we have indicated in 2 , may be mapped into $P$ in the following manner. We map a point of $P^{\prime}$ represented by the class $(u, a), u \in U_{\alpha}, a \in \Psi$, into the point $x=\rho_{\alpha}^{-1}\left(x_{\alpha} \cdot a\right)$ of $P$. This mapping, denoted by $f$, is an injection. We show that $f\left(P^{\prime}\right)$ coincides with the set of points $P_{0}$ which can be joined to $x_{0}$ by a horizontal curve. If we write $x \sim y$ when $x$ and $y$ can be joined by a horizontal curve, then $x_{0} \sim x_{\alpha}$ by the definition of $x_{\alpha}$, and hence $x_{0} a \sim x_{\alpha} a$. Since $a \in \Psi$, we have $x_{n} \sim x_{0} \cdot a$. Then $x=\rho_{\alpha}^{-1} \cdot\left(x_{\alpha} \cdot a\right) \sim x_{\alpha} \cdot a \sim x_{0}$. We have thus shown that any point $f\left(P^{\prime}\right)$ can be joined to $x_{0}$ by a horizontal curve. The converse is also easy to prove.

We shall now show that the given connection in $P$ may be reduced to a connection in the reduced bundle $P_{0}$. If $x \in P_{0}$, then the horizontal subspace $Q_{x}$ of the given connection in $P$ is tangent to $P_{0}$. In fact, any horizontal vector at $x$ is the tangent vector to a certain horizontal curve, which must belong to $P_{0}$ by the definition of $P_{0}$. Thus $x \in P_{0} \rightarrow Q_{x}$ defines a connection in $P_{0}$ and the original connection in $P$ is induced from this connection in $P_{0}$ in the manner of Proposition 1. We have thereby concluded the proof of Proposition 2.

It might be remarked that the holonomy group of $P_{0}$ is exactly $\Psi$.

\section{Holonomy and isotropy groups}

4. A theorem on $\boldsymbol{G} / \boldsymbol{H}$. Let $G$ be a connected Lie group and $H$ a closed subgroup. The homogeneous space $G / H$ may be considered as the base space of a principal fiber bundle $G$ on which $H$ acts to the right in the natural fashion. $G$ acts on $G$ itself to the left.

Proposition 3. In order that there exist a connection in the principal fiber bundle $G(G / H, H)$ which is invariant by the left translations of $G$, it is necessary and sufficient that $G / H$ is reductive in the sense of [11], that is, there is a subspace $\mathfrak{m}$ of the Lie algebra $\mathfrak{g}$ of $G$ such that $\mathfrak{g}=\mathfrak{m}+\mathfrak{h}$ (direct sum), $\mathfrak{h}$ being the subalgebra determined by $H$, and such that $a d(H) \cdot m=m$. More precisely, by taking such a subspace $\mathfrak{m}$ as the horizontal subspace at the identity element $e$ of $G$, we can define an invariant connection in $G$, and vice versa. 
Proof. We regard $g$ as the tangent space of $G$ at the identity element [6]. If there is a connection $\Gamma$ in $G(G / H, H)$, the horizontal subspace at $e$ is a subspace $m$ of $\mathfrak{g}$ such that $\mathfrak{g}=\mathfrak{m}+\mathfrak{h}$, where $\mathfrak{h}$ is considered as the subspace tangent to the fiber $H$ through $e$. By the condition 2) for a connection, we see that the horizontal subspace at a point $h \in H$ is the image of $m$ by the right translation $R_{h}$ of $G$. If $\Gamma$ is invariant by the left translations of $G$, then the horizontal subspace at $h$ must be the image of $m$ by the left translation $L_{h}$. Hence we must have $R_{h} \cdot \mathfrak{m}=L_{h} \cdot \mathfrak{m}$, that is, $\operatorname{ad}(h) \cdot \mathfrak{m}=\mathfrak{m}$. Since this is true for every $h \in H$, we have $\operatorname{ad}(H) \cdot \mathfrak{m}=\mathfrak{m}$.

Conversely, if there exists a subspace $m$ satisfying $\mathrm{g}=\mathrm{m}+\mathfrak{h}$ and $a d(H) \cdot \mathfrak{m}$ $=\mathrm{m}$, we take $\mathrm{m}$ as the horizontal subspace at $e$ in $G$. For any element $a \in G$, the horizontal subspace $Q_{a}$ at a is defined to be the image of $m$ by the left translation $L_{a}$. For any element $h$ of $H$, we have then $R_{h} \cdot Q_{a}=R_{h} \cdot L_{a} \cdot \mathrm{m}$ $=L_{a} \cdot R_{h} \cdot \mathrm{m}=L_{a} \cdot L_{h} \cdot \mathrm{m}=L_{a h} \cdot \mathrm{m}=Q_{a h}$, which is the condition 2) for connections. The condition 3 ) is easy to verify. Proposition 3 is hence proved.

From now on, we assume that $G$ is effective on $G / H$, that is, $H$ does not contain any invariant subgroup $(\neq e)$ of $G$. If furthermore the canonical homomorphism of $H$ onto the linear isotropy group $\tilde{H}$, is one-to-one, then the principal fiber bundle $G(G / H, H)$ admits an injection in the bundle of frames $P(G / H, G L(n, R))$ over $G / H$. Namely, we fix an arbitray frame $x_{0}$ at $P_{0}$ of $G / H$ and map $a \in G$ into the frame which is the image of $x_{0}$ by the transformation of $G / H$ induced by $a$. This mapping $f$ is an injection and is commutative with the transformations of $G$ acting on $G$ to the left and on $P$ in the natural fashion.

If $G(G / H, H)$ admits a connection $\Gamma$ invariant by the left translations of $G$, then the injection $f$ induces, in virtue of Proposition 1 , a connection $\Gamma^{\prime}$ in $P$ which is invariant by $G$. In this way, we obtain an affine connection on $G / H$ invariant by $G$.

Let $X$ be any element of $\mathfrak{m}$ and let $x_{t}$ be the one-parameter group of $G$ generated by $X$. Its tangent vector $x_{t}^{\prime}$ at the point $x_{t}$ is obtained from $X$ by the left translation $L_{x_{t}}$ and belongs to the horizontal subspace at $x_{t}$. Therefore, $x_{t}$ is a horizontal curve in $G$. If $h$ is an arbitrary element of $H$, then the curve $R_{h} \cdot x_{t}=L_{x_{t}} \cdot h$ is also horizontal. Let $\tilde{x}_{t}$ be the image of $x_{t}$ by the canonical projection of $G$ onto $G / H$. Then the parallel displacement along the curve $\tilde{x}_{t}$ 
of the fiber $H$ is the same as the left translation of $H$ by $x_{t}$.

This property of the parallel displacement is transfered to the corresponding invariant affine connection $\Gamma^{\prime}$ on $G / H$. Hence $\Gamma^{\prime}$ is what we have called the canonical affine connection of the second kind on the reductive homogeneous space $G / H[11]$. The torsion and curvature tensor fields being invariant by $G$, they are also invariant by the parallel displacement. This means that their covariant derivatives are zero.

Conversely, we start with a homogeneous space $G / H$ which admits an invariant affine connection. The canonical homomorphism of $H$ onto $\tilde{H}$ is oneto-one [10]. If furthermore the homogeneous holonomy group $\Psi$ is contained in $\tilde{H}$, then the affine connection is reducible, in virtue of Proposition 2 , to a connection in the reduced bundle $P^{\prime}(G / H, \Psi)$ and, a fortiori, to a connection $\Gamma$ in $G(G / H, H)$ which contains $P^{\prime}(G / H, \Psi) . \quad \Gamma$ is certainly invariant by the left translations of $G$. We have thereby proved

Theorem 1. Let $G / H$ be a homogeneous space of a connected Lie group $G$ over a closed subgroup $H$, where $H$ does not contain any invariant subgroup $(\neq e)$ of $G$. If $G / H$ admits an invariant affine connection whose homogeneous holonomy group is contained in the linear isotropy group $\widetilde{H}$, then $G / H$ is reductive and the covariant derivatives of the torsion and curvature tensor fields are all zero.

5. The main theorem. Let $M$ be a Riemannian manifold. We denote by $G$ the largest connected group of isometries of $M$. By the isotropy group $H_{\not}$ at a point $p$ of $M$ we mean the subgroup of $G$ consisting of isometries which leave the point $p$ invariant. The linear isotropy group $\widetilde{H}_{p}$ is the group of linear transformations of the tangent space $T_{p}$ induced by the elements of $H_{p}$. We shall now prove

TheOREM 2. Let $M$ be a complete Riemannian manifold. If the restricted homogeneous holonomy group $\Psi_{p}^{0}$ is contained in the linear isotropy group $\widetilde{H}_{p}$ at each point $p$ of $M$, then $M$ is Riemannian symmetric, that is, the covariant derivatives of the curvature tensor field are zero.

Proof. The proof is divided into three steps.

1) First, we show that it is sufficient to prove the theorem in the case where $M$ is simply connected. Let $\tilde{M}$ be the universal covering manifold 
of $M$ with a natural Riemannian metric induced by the projection $\pi$. Of course, $M$ is symmetric if and only if $\tilde{M}$ is symmetric. We show that the assumption of Theorem 2 remains valid for $\tilde{M}$. The homogeneous holonomy group of $\tilde{M}$ is isomorphic with the restricted homogeneous holonomy group of $M$ in the manner precised in [13]. On the other hand, if $\widetilde{p}$ is any point of $\tilde{M}$ and if $\pi(\tilde{p})=p$, every isometry of $M$ belonging to $H_{p}^{0}$ can be lifted to an isometry of $\tilde{M}$ which belongs to the isotropy group at $\tilde{p}$ of $\tilde{M}$ (Lemma 6 , [13]). This proves our assertion.

2) Assume that $M$ is simply connected and complete. We show that it is sufficient to prove Theorem 2 in the case where $M$ is irreducible. For this purpose, consider the canonical decomposition $M=M_{0} \times M_{1} \times \ldots \times M_{r}$ given by G. de Rham [13], [14]. $M$ is symmetric if and only if every factor $M_{i}$ is symmetric (the Euclidean part $M_{0}$ is trivially so). We show that the assumption of Theorem 2 is valid for each $M_{i}$.

Let $p=\left(p_{0}, p_{1}, \ldots, p_{r}\right) \in M_{0} \times M_{1} \times \ldots \times M_{r}$. The homogeneous holonomy group $\Psi_{p}$ of $M$ is then the direct product $\Psi_{0} \times \Psi_{1} \times \ldots \times \Psi_{r}$ where each $\Psi_{i}$ is the homogeneous holonomy group of $M_{i}$ and acts trivially on $T_{j}$. if $j \neq i$ [13], [14]. On the other hand, let $\varphi$ be any isometry of $M$ belonging to $H_{p}$. Then $\varphi$ leaves invariant each $T_{i}[13]$ and hence induces an isometry $\varphi_{i}$ of $M_{i}$. Obviously, $\varphi_{i}\left(p_{i}\right)=p_{i}$. If we consider the isometry $\psi$ of $M$ defined by $\psi(q)$ $=\left(\varphi_{0}\left(q_{0}\right), \varphi_{1}\left(q_{1}\right), \ldots, \varphi_{r}\left(q_{r}\right)\right)$ for $q=\left(q_{0}, q_{1}, \ldots, q_{r}\right) \in M_{0} \times M_{1} \times \ldots \times M_{r}$, then the linear transformation of $T_{p}$ induced by $\psi$ coincides with the one induced by $\varphi$. Hence $\psi=\varphi$. It is also clear that each $\varphi_{i}$ belongs to the isotropy group $H_{i}$ at $p_{i}$ of $M_{i}$. Hence $H_{p}=H_{0} \times H_{1} \times \ldots \times H_{r}$.

From these considerations, it follows that if $\Psi_{p}$ is contained in $\widetilde{H}_{p}$, then $\Psi_{i}$ is contained in $\widetilde{H}_{i}$ for each $i$. This proves that the assumption of Theorem 2 is valid for each $M_{i}$.

3) Finally, we assume that $M$ is simply connected and irreducible nonEuclidean. The orbit $G(p)$ of $G$ of an arbitray point $p$ is a submanifold of $M$; more precisely, the injection of $G(p)$ provided with the differentiable structure as a homogeneous space $G / H_{p}$ into $M$ is of maximum rank at every point of $G(p)$. Let $T_{p}^{\prime}$ be the subspace of $T_{p}$ tangent to $G(p)$. Since $T_{p}^{\prime}$ is obviously invariant by $\widetilde{H}_{p}$, it is invariant by $\Psi_{p}$ which is irreducible by assumption. Therefore we have two cases: either $T_{p}^{\prime}=(0)$ or $T_{p}^{\prime}=T_{p}$. If $T_{p}^{\prime}=(0)$ at every 
point $p$ of $M$, it means that $G(p)=p$ for every $p . H_{p}$ and hence $\Psi_{p}$ consists of the identity, that is, $M$ is Euclidean. This is contradictory to the assumption.

Hence there exists at least one point $p$ such that $T_{p}^{\prime}=T_{p}$. This means that $G(p)$ contains a neighborhood of $p$ and hence is an open set in $M$. Then $G(p)$ is complete as a Riemannian homogeneous space $G / H_{p}$. Therefore we must have $M=G(p)=G / H_{p}$. We conclude the proof of Theorem 2 by applying Theorem 1.

Remark. In Theorem 2, it is sufficient to assume that the homogeneous holonomy group is contained in the linear isotropy group at each point of a (non-empty) open set $U$ of $M$. Indeed, in the above proof, we have only to check the following point in 3$)$. If $T_{p}^{\prime}=(0)$ at every point $p$ of $U$, then $G(p)=p$ in $U$. Since any isometry which is the identity on an set is the identity on the whole manifold, we see that $G$ consists of the identity only.

6. Applications. Let $M$ be a complete Riemannian manifold of dimension $n$. Let $r$ and $s$ be the dimension of the largest connected group of isometries $G$ and that of the restricted homogeneous holonomy group $\Psi_{p}^{0}$, respectively.

THEOREM 3. Let $M$ be a complete Riemannian manifold whose restricted homogeneous holonomy group is irreducible. If $r=s+n$, then either $M$ is $a$ Kählerian manifold whose Ricci curvature is zero or $M$ is symmetric.

Proof. Let $p$ be an arbitray point of $M$ and let $H_{p}$ be the isotropy group at $p$. $G / H_{p}$ being the orbit of $p$ by $G$, we have $r-\operatorname{dim} H_{p}^{0} \leqq n$. On the other hand, if $M$ is not a Kählerian manifold whose Ricci curvature is zero, the connected component of the normalizor of $\Psi_{p}^{0}$ coincides with $\Psi_{p}^{0}$ [9]. Therefore, $\widetilde{H}_{p}^{0}$ is contained in $\Psi_{p}^{0}$ and hence $\operatorname{dim} \widetilde{H}_{p}^{0} \leqq s=r-n$. From these two inequalities, we get $\operatorname{dim} \widetilde{H}_{p}^{0}=r-n=\operatorname{dim} \Psi_{p}^{0}$ and hence $\widetilde{H}_{p}^{0}=\Psi_{p}^{0}$. This holds at every point $p . \quad M$ is then symmetric in virtue of Theorem 2 .

For any Riemannian manifold whose $\Psi_{p}^{0}$ is irreducible and which is not a Kählerian manifold with Ricci curvature equal to zero, we have always $r \leqq s+n$ [8]. Theorem 3 gives another characteristic property of symmetric spaces among the class of Riemannian manifolds in consideration.

THEOREM 4. Let $M$ be a compact Riemannian manifold whose restricted homogeneous holonomy group is irreducible. If $r=s+n$, then $M$ is symmetric. 
Proof. We have only to show that the Ricci curvature is not zero. If it is zero, then the covariant derivatives of any Killing vector field are zero [2]. Since $M$ is irreducible, such a vector field is zero. This means that $G$ consists of the identity only and $r=0$, which is contradictory to the assumption $r=s+n$.

\section{BIBLIOGRAPHY}

[1] W. Ambrose and I. Singer, A theorem on holonomy, Trans. Amer. Math. Soc., 75 (1953), 428-443.

[2] S. Bochner, Vector fields and Ricci curvature, Bull. Amer. Math. Soc., 52 (1946), 776797.

[ 3 ] E. Cartan, Les groupes d'holonomie des espaces généralisés, Acta Math., 48 (1926), 1-42.

[4] E. Cartan, Sur une classe remarquable d'espaces de Riemann, Bull. Soc. Math. France, 54 (1926), 214-264, and 55 (1927), 114-134.

[ 5 ] S. S. Chern, Topics in differential geometry, Institute for Advanced Study, 1951.

[6] C. Chevalley, Theory of Lie groups I, Princeton 1946.

[ 7 ] C. Ehresmann, Les connexions infinitésimales dans un espace fibré différentiable, Colloque de Topologie, Bruxelles (1950), 29-55.

[8] S. Kobayashi, Des groupes linéaires irréductibles et la géométrie différentielle, Proc. Japan Acad., 30 ( 1954), 934-936.

[ 9 ] A. Lichnerowicz, Espaces homogènes kählériens, Colloque de Géométrie Différentielle, Strasbourg (1953), 171-184.

[10] K. Nomizu, On the group of affine transformations of an affinely connected manifold, Proc. Amer. Math. Soc., 4 (1953), 816-823.

[11] K. Nomizu, Invariant affine connections on homogeneous spaces, Amer. Journ. Math., 76 (1954), 33-65.

[12] K. Nomizu, Remarques sur les groupes d'holonomie et d'isotropie, Colloque de Topologie, Strasbourg (mai, 1954).

[13] K. Nomizu, Studies on Riemannian homogeneous spaces, in this journal.

[14] G. de Rham, Sur la réductibilité d'un espace de Riemann, Comment. Math. Helv., 26 (1952), 328-244.

[15] N. Steenrod, The topology of fiber bundles, Princeton, 1951.

Mathematical Institute, Nagoya University 